\title{
Precision Measurement Of The Neutron's Beta Asymmetry Using Ultra-Cold Neutrons
}

\author{
Makela, M. ${ }^{1}$, Back, H.O. ${ }^{2}$, Melconian, D. ${ }^{3}$, Plaster, B. ${ }^{4}$, et al. \\ ${ }^{1}$ Los Alamos National Lab., P.O. Box 1663, Los Alamos, NM 87545; ${ }^{2}$ North Carolina State \\ University Raleigh, NC 27695; ${ }^{3}$ University of Washington, Department of Physics, Box 351560, \\ Seattle, WA 98195; ${ }^{4}$ California Institute of Technology, Kellogg Radiation Lab, Pasadena, CA 91125
}

\begin{abstract}
A measurement of $A_{\beta}$, the correlation between the electron momentum and neutron (n) spin (the beta asymmetry) in $\boldsymbol{n}$ beta-decay, together with the $\boldsymbol{n}$ lifetime, provides a method for extracting fundamental parameters for the charged-current weak interaction of the nucleon. In particular when combined with decay measurements, one can extract the $\mathrm{V}_{\mathrm{ud}}$ element of the CKM matrix, a critical element in CKM unitarity tests. By using a new $\mathrm{SD}_{2}$ super-thermal source at LANSCE, large fluxes of UCN (ultra-cold neutrons) are expected for the UCNA project. These UCN will be $100 \%$ polarized using a $7 \mathrm{~T}$ magnetic field, and directed into the $\beta$ spectrometer. This approach, together with an expected large reduction in backgrounds, will result in an order of magnitude reduction in the critical systematic corrections associated with current $\boldsymbol{n} \beta$-asymmetry measurements. This paper will give an overview of the UCNA $\mathrm{A}_{\beta}$ measurement as well as an update on the status of the experiment.
\end{abstract}

Keywords: Neutron beta asymmetry, CKM unitarity, ultra-cold neutron source PACS: 12.15.-y, 12.15.Hh, 14.20.Dh, 23.40.-s 29.25.Dz, 23.20.En

\section{Introduction - Beta Decay and Ultra-cold Neutrons}

Experiments with polarized ultra-cold neutrons (UCN) offer a new way to measure the decay correlations of neutron beta decay; these correlations can be used to test the completeness of the Standard Model and constrain new models beyond it. Ultra-cold neutrons are very low energy neutrons $(<300 \mathrm{neV})$ that can be trapped inside of material and magnetic bottles. The decay correlations in combination with the neutron and muon lifetimes experimentally determine the first element $\left(\mathrm{V}_{\mathrm{ud}}\right)$ of the CabibboKobayashi-Maskawa (CKM) quark mixing matrix. The CKM matrix is a unitary transform between the mass and weak eigenstates of the $\boldsymbol{d}, \boldsymbol{s}$ and $\boldsymbol{b}$ quarks; if the matrix is not unitary this would imply that the Standard Model is not complete. Currently the unitarity of the first row of the CKM matrix is in question and $V_{u d}$ is the largest component of the row. $1,2,3$

Precision measurements of $\mathrm{V}_{\mathrm{ud}}$ are obtained from two different types of experiments: the decay correlation between the electron and the polarized neutron with the lifetime in neutron beta decay, and the super-allowed $0^{+} \rightarrow 0^{+}$nuclear beta decays (both of these must be combined with $\mathrm{G}_{\mathrm{F}}$ from muon decay). The super-allowed $0^{+} \rightarrow 0^{+}$decays currently give the most accurate values of $\mathrm{V}_{\mathrm{ud}}$, but theoretical errors dominate the uncertainty. 1, 2, 3,4 Neutron beta decay has lower theoretical uncertainty but the experimental errors are great and the various experiments do not agree with

CP842, Particles and Nuclei,

Seventeenth International Conference on Particles and Nuclei

edited by P. D. Barnes, M. D. Cooper, R. A. Eisenstein, H. van Hecke, and G. J. Stephenson

(C) 2006 American Institute of Physics 0-7354-0338-4/06/\$23.00

808 
each other. The goal of the UCNA experiment (an experiment to measure the Acorrelation using UCN) is to lower the experimental error in $\mathrm{V}_{\mathrm{ud}}$ obtained from neutron beta decay so that it is comparable to that from super-allowed $0^{+} \rightarrow 0^{+}$decays. The UCNA experiment will be the first measurement of the A-correlation of polarized neutron beta decay using UCN; the previous precision measurements were made with cold neutron beams at reactors.

The UCNA experiment will be a complete departure from previous cold neutron experiments with different and lower backgrounds and corrections to be applied in the extraction of the A-correlation. The two main advantages of the UCNA experiment are $100 \%$ polarization of the neutrons and a pulsed beam induced prompt background. The neutron source is a pulsed spallation target, rather than a reactor, so the backgrounds are time dependent and very different than previous reactor based cold neutron beam experiments. The $100 \%$ polarization of the neutrons is achieved by passing the UCN through a seven Tesla magnetic field; one spin-state is blocked by the interaction of the neutron's magnetic moment with the field, while the other is accelerated through it. In order to have high polarization in the experiment the UCN must maintain polarization until they decay or leave the decay region. The UCN travel a couple of meters and bounce off the guide walls many times between the polarizer and the decay region; each of these wall interactions can potentially depolarize the UCN.

\section{The UCNA Experiment}

The UCNA experiment has a dedicated source, state-of-the-art guides, high efficiency polarization and spinflipping, $4 \pi$ beta collection, low-backscatter beta detection and low backgrounds. All of these features combine to provide a new approach to measuring the A-correlation of polarized neutron beta decay with minimal error due to uncertainties in polarization and background. The UCNA neutron source is a super thermal solid deuterium UCN source. 5, 6, 7

The source for the UCNA experiment is a scaled up version of a prototype source that achieved a world-record density of greater than $100 \mathrm{UCN}$ per cubic centimeter. Neutrons are provided by a pulsed proton spallation source. ${ }^{8,9}$ These neutrons are trapped, elastically cooled, and finally down-scattered in the solid deuterium to become UCN. Due to the intense radiation of the interacting proton beam, the source is located inside a steel and concrete crypt; the UCN must be guided out of this crypt and into the experimental area.

The guide system is made with low depolarization, state-of-the-art diamond-like carbon coated quartz guide tubes. There are about 12 meters of guide before the UCN enter the decay region of the experiment. Even with the high quality guides only about $1 \%$ of the UCN reach the decay region. Before entering the decay region the UCN are polarized with a 7 tesla solenoidial magnetic field. This field provides $100 \%$ initial polarization of the UCN. The magnetic field is tailored on the downstream side of the magnetic field where the UCN spinflipper is located.

The spinflipper is of the adiabatic fast passage type, which has been shown to be over $99.9 \%$ efficient. This high spinflipping efficiency combined with $100 \%$ polarization provides the highest polarization ever used in an A-correlation 
experiment. The diamond coated quartz guides and magnetic holding fields are needed to keep the UCN polarized while they travel into the decay region.

The decay region is a three-meter-long, ten-centimeter-diameter diamond-coated quartz tube that the UCN enter in the middle. To give $4 \pi$ collection of the decay electrons this tube is inside a 1 Tesla solenoidial magnetic field. Electron detectors at the ends of the decay region detect the beta particles. These detectors combine low backscatter wire chambers and energy monitoring plastic scintillators. The Acorrelation is calculated from the difference in counts between the detectors at each end of the decay region.

Backgrounds are minimal at the detectors and have a time structure due to the pulsing of the shielded spallation target. Cosmic-ray backgrounds are minimized by surrounding the decay region with plastic scintillator. UCN that do not decay in the decay region are trapped on lithium fluoride surfaces that cause only a low energy gamma background.

\section{Precision and uncertainty in UCNA}

The goal of the UCNA experiment is to make a $0.2 \%$ measurement of the Acorrelation with minimal or no correction due to systematic effects. The main systematic effects are neutron polarization, and betas that backscatter off the detectors; these two effects combine to make up most of the $1.7 \times 10^{-3}$ systematic effect in the measured value of the A-correlation. This systematic effect is below the $2.4 \times 10^{-3}$ systematic effect corrected for in the PERKEO II experiment. ${ }^{10}$ By making accurate measurements of these systematic effects (total error in measurement $4.2 \times 10^{-4}$ ) they can be separated from the measured $\mathrm{A}_{0}$ value to give an even more accurate value. $\mathrm{A}$ large amount of work has already gone into understanding these systematic errors with offline experiments and Monte Carlo work. As a result these projected errors seem obtainable.

A $0.2 \%$ measurement of the A-correlation combined with the current $0.1 \%$ measurement of the neutron lifetime will give a $0.06 \%$ measurement of $V_{u d}$ which is comparable to the $0.05 \%$ error in $\mathrm{V}_{\mathrm{ud}}$ from super-allowed $0^{+} \rightarrow 0^{+}$nuclear beta decays and $>.06 \%$ error from demanding unitarity using various values of $\mathrm{V}_{\mathrm{us}}$ and $\mathrm{V}_{\mathrm{ub}}$.

\section{REFERENCES}

1. Abele, H. et al., European Physical Journal C 33, 1-8 (2004).

2. Czarnecki, A., Marciano, W. J., Sirlin, A., Physical Review D 70, p.093006 (2004).

3. Hardy, J. C., Towner, I. S., Physical Review Letters 94, p.092502/1-4 (2005).

4. Eidelman, S. et al., Physics Letters B592, p.093006 (2004).

5. Golub, R., Richardson, D. and Lamoreaux, S. K., Ultra-Cold Neutrons, Bristol, Philadelphia, New York: Adam Hilger, 1991, pp. 62-77.

6. Ignatovich, V. K., The Physics of Ultracold Neutrons, Oxford: Clarendon Press, 1990, pp. 31-37.

7. Hill, R. E. et al., NIM A 440, 674-681 (2000).

8. Morris, C. L. et al., Physical Review Letters 89, pp 272501/1-4 (2002).

9. Saunders A. et al., Physics Letters B 593, 55-60 (2004).

10. Abele, $\mathrm{H}$ et al., "The Beta-, Neutrino- and Proton-Asymmetry in Neutron b-Decay" in International Conference on Precision Measurements with Slow Neutrons 2004, J. Res. Inst. Stand. Technol. 110, Gaithersburg, MD: Natl Inst Stand \& Technol;, 2005, pp. 377-381. 